\title{
The effect of direct-acting antivirals on hepatocellular carcinoma recurrence: still waiting for the turning point
}

\author{
Gaetano Serviddio, Rosanna Villani \\ Liver Unit, Centro Universitario per la Ricerca e la cura delle Epatopatie (C.U.R.E.), Department of Medical and Surgical Sciences, University of \\ Foggia, Foggia, Italy \\ Correspondence to: Professor Gaetano Serviddio. University Centre for Liver Disease Research and Treatment, Department of Medical and Surgical \\ Sciences, University of Foggia, 71122 Foggia, Italy. Email: gaetano.serviddio@unifg.it. \\ Comment on: Singal AG, Rich NE, Mehta N, et al. Direct-Acting Antiviral Therapy Not Associated With Recurrence of Hepatocellular Carcinoma in \\ a Multicenter North American Cohort Study. Gastroenterology 2019;156:1683-92.e1.
}

Submitted May 07, 2019. Accepted for publication May 16, 2019.

doi: 10.21037/hbsn.2019.05.09

View this article at: http://dx.doi.org/10.21037/hbsn.2019.05.09

Hepatocellular carcinoma (HCC) is the fifth most common cancer worldwide accounting for about $9 \%$ of all cancer deaths (1). The natural history of HCC is extremely variable due to high effectiveness of treatment only in early stages and low overall survival rate in patients with advanced disease who are poor responders to any treatment. Unlike other cancers, HCCs usually develop in patients with underlying liver disease that limits the use of chemotherapy and radiotherapy for the high risk of complications (2).

$\mathrm{HCV}$ is the main cause of chronic liver disease, cirrhosis and HCC in the world (3). In patients with chronic $\mathrm{HCV}$ infection, the annual HCC incidence is up to $8.8 \%$ in untreated patients with liver cirrhosis (4). Therefore, in the context of persistent HCV-related hepatitis, the key goals of antiviral therapy are to treat the infection, to reduce transmission rate and, especially, HCC incidence.

The direct-acting antivirals (DAAs) have revolutionized the management of patients with HCV infection because of their efficacy and safety profile even in populations considered ineligible to IFN therapy.

Unfortunately, several authors have reported an unexpected high rate of HCC recurrence in patients treated with DAAs (5).

Very recently, Marino et al. showed an increased risk of HCC recurrence in patients treated with interferon-free regimens in the largest European cohort study of patient treated with DAAs (1,123 patients) (6).

On the contrary, Singal et al. have just published the results of a large retrospective analysis of a multicentre North American cohort study including 304 HCV-related
HCC patients treated with DAAs vs. 489 untreated patients. In DAA-treated group, HCC recurrence was observed in $42.1 \%$ of patients as compared to $58.9 \%$ of untreated group, without a statistically significant difference.

Even if the study tried to put an end to the debate on the risk of DAAs on HCC recurrence, many factors should still be considered.

The number of cases of recurrence in untreated patients reported by Singal was dramatically higher $(42.1 \%$ and $58.9 \%)$ than that previously reported by other authors in cirrhotic patients $(5,7)$, probably due to a more advanced liver disease and worse clinical conditions.

The two populations included in the analysis were significantly different in terms of HCC treatment, number of treatments to achieve tumor response, and Child Pugh class.

DAA treated patients were more frequently treated with curative therapy (resection or local ablation) whereas most of untreated patients underwent trans arterial chemoembolization (TACE).

Even if DAA treated patients had better clinical conditions at baseline they showed a significant higher risk of HCC development compared to patients treated with interferon who have an annual occurrence rate of $1.4 \%$ after SVR $(8,9)$ and HCC patients showing a recurrence in up to $35 \%$ in respect of baseline characteristic of HCC (10).

In their study, HCC complete response to any treatment was established after a median time of 1.6 months and a median follow-up time to exclude HCC recurrence was 10.4 months. Data from the literature show a grow rate 
ranging from 1 month to 1.3 years $(11,12)$ suggesting that HCC surveillance program require a long-term follow-up before excluding tumor recurrence. It is conceivable that many cases were not HCC recurrence but rather HCC persistence.

The time to HCC recurrence is strategic when we consider the potential mechanisms accounting for the HCC reappearance. We have suggested that DAAs may promote the development of new liver nodules only in patients with residual HCC cells by eliciting immune-related mechanisms or by increasing serum VEGF level which could promote tumor growth and, finally, recurrence (13). In this context, patients with complete response after tumor treatment who do not have residual tumor cells would not develop tumor recurrence even if exposed to high VEGF level. In our clinical experience, we usually treat HCC patients by DAAs only after 6-9 months of negative surveillance and from there we have not observed any further case of HCC recurrence.

Singal et al. makes a significant contribution because of the large population studied and data collection. However, the shorter follow-up and the potential inclusion of patients with residual disease in the analysis limit the final conclusion.

The debate is still on and the turning point seems to be far enough.

\section{Acknowledgments}

None.

\section{Footnote}

Conflicts of Interest: The authors have no conflicts of interest to declare.

\section{References}

1. Wong MC, Jiang JY, Goggins WB, et al. International incidence and mortality trends of liver cancer: a global profile. Sci Rep 2017;7:45846.

2. Pinter $M$, Trauner $M$, Peck-Radosavljevic $M$, et al. Cancer and liver cirrhosis: implications on prognosis and management. ESMO Open 2016;1:e000042.

3. de Oliveria Andrade LJ, D'Oliveira A, Melo RC, et al. Association between hepatitis $\mathrm{C}$ and hepatocellular carcinoma. J Glob Infect Dis 2009;1:33-7.
4. Xu F, Moorman AC, Tong X, et al. All-Cause Mortality and Progression Risks to Hepatic Decompensation and Hepatocellular Carcinoma in Patients Infected With Hepatitis C Virus. Clin Infect Dis 2016;62:289-97.

5. Reig M, Marino Z, Perello C, et al. Unexpected high rate of early tumor recurrence in patients with HCV-related HCC undergoing interferon-free therapy. J Hepatol 2016;65:719-26.

6. Marino Z, Darnell A, Lens S, et al. Time association between hepatitis $\mathrm{C}$ therapy and hepatocellular carcinoma emergence in cirrhosis: relevance of non-characterized nodules. J Hepatol 2019;70:874-84

7. Conti F, Buonfiglioli F, Scuteri A, et al. Early occurrence and recurrence of hepatocellular carcinoma in HCVrelated cirrhosis treated with direct-acting antivirals. J Hepatol 2016;65:727-33.

8. El-Serag HB, Kanwal F, Richardson P, et al. Risk of hepatocellular carcinoma after sustained virological response in Veterans with hepatitis $\mathrm{C}$ virus infection. Hepatology 2016;64:130-7.

9. Yu ML, Lin SM, Chuang WL, et al. A sustained virological response to interferon or interferon/ribavirin reduces hepatocellular carcinoma and improves survival in chronic hepatitis $\mathrm{C}$ : a nationwide, multicentre study in Taiwan. Antivir Ther 2006;11:985-94.

10. Singal AK, Freeman DH Jr, Anand BS. Meta-analysis: interferon improves outcomes following ablation or resection of hepatocellular carcinoma. Aliment Pharmacol Ther 2010;32:851-8.

11. Kubota K, Ina H, Okada Y, et al. Growth rate of primary single hepatocellular carcinoma: determining optimal screening interval with contrast enhanced computed tomography. Dig Dis Sci 2003;48:581-6.

12. Sheu JC, Sung JL, Chen DS, et al. Growth rate of asymptomatic hepatocellular carcinoma and its clinical implications. Gastroenterology 1985;89:259-66.

13. Villani R, Facciorusso A, Bellanti F, et al. DAAs Rapidly Reduce Inflammation but Increase Serum VEGF Level: A Rationale for Tumor Risk during Anti-HCV Treatment. PLoS One 2016;11:e0167934.

Cite this article as: Serviddio G, Villani R. The effect of direct-acting antivirals on hepatocellular carcinoma recurrence: still waiting for the turning point. HepatoBiliary Surg Nutr 2019;8(5):525-526. doi: 10.21037/hbsn.2019.05.09 